schen Ziele von DNZ und DVU erscheinen kaum als diskussionsfähig. Der Grund ist: DNZ und DVU werden in der Nähe des Nationalsozialismus gesehen. Der Nationalsozialismus aber war eine Bewegung, die sich gegen die Orientierung der Gesellschaft an Inklusion (allgemeine Menschengleichheit) und Funktion (Problemlösungsvergleich) richtete ${ }^{8}$, wie sie bereits Art. I der französischen Erklärung der Rechte des Menschen und des Bürgers von 1789 formuliert hatte: Frei und gleich an Rechten werden die Menschen geboren und bleiben es. Die sozialen Unterschiede können sich nur auf den gemeinsamen Nutzen gründen.« Inklusion und Funktion verhalten sich prekär zueinander. Funktionsorientierung ist nur auf der Basis von Gleichheit möglich und schafft zugleich Ungleichheiten. Beide bestimmen jedoch die Identität der modernen Gesellschaft. Damit die moderne Gesellschaft existieren kann, muß sie beide Prinzipien moralisieren. Wenn man aus diesem Tatbestand eine ethische Regel ableiten will, müßte sie lauten: wir sollen mit offenen Fragen und Widersprüchen leben.

\title{
Günter Frankenberg Vom Schweigen der Öffentlichrechtler und ihrer Verantwortung, dieses bisweilen zu brechen
}

\section{Nachwort}

Facta sunt servanda. Der Staatsrechtslehrer M. verstirbt 1993 im biblischen Alter von 92 Jahren. Er nimmt eine bewegte Vergangenheit mit ins Grab: Bayerischer Staatsdienst zu Zeiten der Weimarer Republik; bereits ab 1934 argumentative Dienstleistungen für das NS-Regime; 1935 Professor für Staats- und Verwaltungsrecht und Teilnehmer an einem NS-Dozentenlager; 1948 Mitwirkung im Verfassungskonvent von Herrenchiemsee; 1952 Berufung an die Universität München, begleitet von studentischen Protesten; daneben ab 1957 Bayerischer Staatminister für Unterricht und Kultus, Rücktritt wegen seiner Vergangenheit auf Drängen der parlamentarischen Opposition; fortan nur noch, aber ungeheuer produktiver Staatsrechtslehrer. Schließlich nach seinem Tode die Enthüllung, er habe bis zum letzten Atemzug, unter dem Deckmantel der Anonymität, eine der führenden Figuren der neonationalsozialistischen Szene juristisch beraten.

Nicht die Person ${ }^{1}$, wohl aber der Öffentlichrechtler und sein Werk ${ }^{2}$ geraten - wieder

8 Gerd Roellecke, Der Nationalsozialısmus als politisches Layout der Bundesrepublik Deutschland, Staat 28 (1989), S. 504-524, 517; zu Entsprechungen im Fall des Kommunismus vgl. derselbe, Sozialismus und deutsche Wiedervereinigung, Staat 29 (1990) S. 481-496, $488 \mathrm{ff}$

I Dem Menschen M. bestreiten auch die Kritiker nicht Gute, Fleiß, Toleranz und andere positive Eigenschaften. Roelleckes Behauptung (stehe »Theodor Maunz und die Verantworcung des Óffentlichrechtlers", in diesem Heft), die "gesamte Person " werde (nicht: habe sich) diskreditiert, ist, mit Verlaub, blanker Unsınn. Vgl. Stolleis, Theodor Maunz - Eın Staatsrechtslehrerleben, KJ 1993, $393 \mathrm{ff.,} \mathrm{dem} \mathrm{es} \mathrm{um} \mathrm{die}$ Verantwortung des Öffentlichrechtlers geht. Auch Deiseroth, Kontinuitaitsprobleme der deutschen Staatsrechtslehre(r)? Das Beispiel Theodor Maunz, in: Ordnungsmacht? Uber das Verhaltnis von Legalitat, Konsens und Herrschaft, hrsg. v. Deiseroth, Hase und Ladeur (Frankfurt am Main 1981), 85 ff., erhebt gegen die Person keine Einwände, wohl aber gegen den Lehrer und Autor des Staatsrechts. Gleiches gilt fur Redeker, Bewaltigung der Vergangenheıt als Aufgabe der Justiz, NJW 1964, $1097 \mathrm{ff}$. und Hollerbach, Jurıstische Lehre und Forschung in Freiburg in der Zeit des Natıonalsozialismus, in: John (Hg.), Die Freiburger Universitat in der Zeit des Nationalsozialismus (1991), 104.

2 Dazu Redeker, (Fn. 1). Eın Schriftenverzeichnis fur die Arbeiten bis 1945 findet sıch beı Haney, Maunz im 
- ins Zwielicht. Wurden eben noch seine »unmittelbar Sachzugewandtheit" und gestaltungskräftige »Nüchternheit«, die »Achtung des anderen Menschen« und " $\mathrm{Zu}$ neigung zu praktischer Gestaltung « gepriesen ${ }^{3}$, dabei die staatsrechtlichen Dienste für das nationalsozialistische Regime schamhaft als »wechselhafte Erfahrung « schöngeredet und Brüche oder Kontinuitäten nach 1945 nicht einmal thematisiert, ${ }^{4}$ so erinnert man sich nun daran, daß Hannah Arendt I 95 I unter anderem auch M. als eine der "zweit- und drittrangigen Begabungen « kennzeichnete, derer sich »die Nazis « als Schmitt-Ersatz bedienten.' Und fragt sich, wie es denn kam, daß diese Begabung nach 1945 zu den meistzitierten Autoren im Verfassungs- und Verwaltungsrecht aufsteigen konnte und der braunen Bewegung - etwa durch Rechtsgutachten zum Parteienrecht - erneut zu Diensten war. Fleiß allein, M.'s Verleger spricht von "Vielschreiberei«, dürfte als Erklärung ebensowenig hinreichen wie »asiatische Höflichkeit (Dürig), die angeblich das Nein-sagen erschwert. ${ }^{6}$ Die genaue Antwort, ohnehin nicht zweifelsfrei zu ermitteln, kann dahingestellt bleiben. Denn M. kann sich nicht mehr äußern; und seinem Mandanten, dem »sehr verehrten, lieben Herrn Doktor Frey « ${ }^{7}$, sollte die Rolle des Kronzeugen verweigert werden. M. ist kein Fall mehr. Dazu fehlt ihm auch das Überraschungsmoment. Wer hätte, angesichts seiner Karriere, diese letzte Wendung für gänzlich unmöglich gehalten? So wird der Fall zum Anlaß, sich Gedanken zu machen über Verantwortlichkeit und Selbstverständnis der Öffentlichrechtler, das sich beim Anlaß M. in breitem öffentlichen Schweigen äußerte. Nur punktuell unterbrochen von Stimmen, die überwiegend Anstoß nahmen. Freilich nicht an M., sondern an Stolleis, der M. zu kritisieren wagte.

\section{Nachruf}

De mortuis nihil nisi bene. Heißt wörtlich übersetzt: Was immer über einen Toten gesagt werde, möge gut, sprich: wohl formuliert, gesagt werden. Eben nicht: de mortuis nihil nisi bonum. In einigen Reaktionen auf die Enthüllung der Karriere von M. wird daraus gleichwohl ein Gebot, nur Gutes zu sagen, jedenfalls das Schlechte, wenngleich Zutreffende gnädig zu bemänteln. ${ }^{8}$ Wer das, was war und wahr ist, ausspricht, wirft folglich "Steine auf ein Staatsrechtler-Grab «. ${ }^{9}$ Von links, versteht sich. Nur: lateinisch lassen sich solche Pietät \& Takt nicht begründen. Wohl deshalb bricht der Enthüllungskritiker Kaiser nach dem »De mortuis ... « ab.

Oder sollte die »bedenkliche « Tätigkeit ${ }^{\circ \circ}$ des Öffentlichrechtlers, weil anonym geschehen, unter einem verlängerten Anonymitätsvorbehalt stehen? Wer nicht M.'s Beratungshilfe für Neonazis, doch deren Heimlichkeit lobt, weil sie, wäre sie geheim geblieben, keinen Schaden angerichtet hätte, weil M. den Rechtsradikalen sein öffentliches Ansehen nicht zur Verfügung stellte, mag immerhin bedenken, daß (Neo-)Nazis, damals wie heute, zur Anonymität ein strategisches Verhältnis unter-

\footnotetext{
Dienste des Faschısmus und der CSU. Eine Dokumentation, hrsg. v. d. Rechtswissenschaftlichen Fakultat der Friedrich-Schiller-Universitat Jena (Jena 1964$), 72 \mathrm{ff}$.

3 Lerche, Theodor Maunz, AoR 1994, i 6 f. und ders., Theodor Maunz, in: Juristen im Portrait. Festschrift zum 22 sjahrigen Jubılaum des Verlages C. H. Beck (Munchen 1988), $553 \mathrm{ff}$.

4 Ansatze dazu finden sich bei Deiseroth, (Fn. I).

5 Elemente und Ursprunge totaler Herrschaft 1951 (Munchen 1986), 544.

6 Vgl. dazu Kuhnert, Maunz raus?, DIE ZEIT v. 10. 2. 1994, S. 66

7 So die von M. gewahlte briefliche Anrede, abgedruckt in Faksımile in: DNZ v. 24. 9. 1993; zit. auch in SZ v. 12./13.2.1994.

8 E.g. Lerche, Theodor Maunz, AoR 1994, $156 \mathrm{f}$

9 J.H. Kaiser in einem Leserbrief, FAZ v. 29. I. 1994.

to So auch K. Vogel, Leserbrief, FAZ v. 5. I. 1994, Nr. 3.
} 

bungsverfahrens scheuen. M. wußte das nach 1933 flink zu rechtfertigen. ${ }^{11}$

\section{Verrat?}

Wenn also die anonyme Tat schwerlich um ihrer selbst willen Anonymität verdient, so könnte sich doch für die darob entsetzten Öffentlichrechtler (die anderen schweigen ohnehin) aus anderen Gründen eine Pflicht zum Beschweigen des Entsetzlichen ergeben. Roellecke impliziert das, wenn er im Kontext seiner Drittschadensliquidation konstatiert, wer den Fehler eines Kollegen "öffentlich breittritt«, verhalte sich »unkollegial«. Und Stolleis, von Roellecke nicht mit offenem Visier attackiert, wäre »ein charakteristischer Fall von Illoyalität«, und vom Kollegium, so die perfide Suggestion, mit Achtungsentzug zu bestrafen. ${ }^{12} \mathrm{Zu}$ befürchten ist, daß Roellecke in diesem Punkt recht behalten könnte, wenn das öffentliche Schweigen der Öffentlichrechtler andauert.

Eine Schweigepflicht aus Kollegialität oder Loyalität? Loyalität ist eine passive Tugend. Strukturell paßt sie zum Schweigen. Ihre Verletzung gilt als Verrat. Wen aber hätten diejenigen Öffentlichrechtler verraten, die den Fall des Öffentlichrechtlers M. publik machten? Ihre Mitgliedschaften müßten darüber Auskunft geben, denn nur aus ihnen kann Loyalität sich herleiten. ${ }^{13}$ Abgesehen von seinem ${ }^{14} \gg$ Auch-Mensch $\ll-$ Sein ist ein Öffentlichrechtler vor allem Beamter, Hochschullehrer, Wissenschaftler, Staatsrechtslehrer, Staatsangeöriger, Gesellschaftsmitglied und, nicht zuletzt, Mitglied einer Gemeinschaft moralischer Wesen. Der Reihe nach. Fragt man, wem oder was ernsthaft eine besondere Loyalitätspflicht geschuldet sein könnte, so scheidet die Gesellschaft aus. Ihre legitimen Erwartungen an Öffentlichrechtler, die sie sich im öffentlichen Dienst hält und von denen sie Dienstleistungen einfordert, werden von den Vorschriften des Beamten- und Hochschulrechts bedient. Eine Schweigepflicht mit Drittwirkung ist diesen Normen nicht zu entnehmen. Für den Staat, obwohl in Sachen politischer Loyalität hierzulande traditionell nicht wenig anspruchsvoll, läßt sich selbst bei großzügiger Auslegung der ohnehin über die Maßen ausgedehnten Treuepflicht im »Radikalenbeschluß «'s keine Pflicht begründen, Verfehlungen von Kollegen zu beschweigen. Viel weniger kann das mithin für den Dreh- und Angelpunkt der Loyalitätspflicht von Öffentlichrechtlern gelten - ihre Bindung an die Verfassung.

Bleibt die Loyalitätspflicht gegenüber der scientific community, präziser: der Vereinigung deutscher Staatsrechtslehrer zu prüfen. Hier verschmilzt Loyalität mit Kollegialität; und die Konturen einer Rechtspflicht verschwimmen in den trüben Fluten eines mehr als fragwürdigen Berufsethos. Das Beschweigen kollegialer Fehltritte, glaubt man den Kritikern der Kritiker von M., wäre geradezu eine sittliche Leistung, beschützte es doch die »Vertrauenswürdigkeit « der Korporation und sicherte damit die Bereitschaft des Publikums, das von Öffentlichrechtlern Gesagte und Geschriebene als wahr abzunehmen, genauer wohl: für bare Münze zu nehmen. ${ }^{16} \mathrm{Ob}$

I I Ausfuihrlich dazu Redeker, (Fn, 1)., I089ff.

12 Roellecke, (Fn. I)

13 Vgl. Fletcher, Loyalitat (Frankfurt am Main 1994), passim.

I4 Eine geschlechtsneutrale Formulierung wurde Geist und Erscheinungsbild der Vereinigung deutscher Staatsrechtslehrer verfehlen. Es ware erfreulich, könnte in absehbarer Zeit ohne Wenn und Aber in gleıcher Weise auch von der Verantwortung der Öffentlıchrechtlerin gesprochen werden.

is BVerfGE $39,334 \mathrm{ff}$.

16 Roellecke: »An der Vertrauenswürdıgkeit und am Ansehen der Organısation haben alle Mitglieder Interesse, weil beides dic Akzeptanz von Entscheidungen erleichtert und das Ansehen jedes Mitgliedes mehrt. « (a. a. O.) Vielleicht erklart dieser verraterische Satz das Schweigen auch der jungeren Öffentlichrechtler in Sachen $M$. 
allerdings eine Vereinigung von Öffentlichrechtlern (die hartnäckig an der traditionellen Bezeichnung "Staatsrechtslehrer « festhält), die im Ruf stünde, ein Schweigekartell zu sein, in einer republikanischen Umgebung Vertrauen genießen könnte, läßt sich empirisch mit guten Gründen bezweifeln. Auch normativ spricht einiges dafür, von einer nach Rekrutierung, Selbstverständnis und Berufspraxis eher konservativen und ideologieanfälligen Berufsgruppe mit prekärer Herrschaftsaffinität zu fordern, sie möge ihren Korpsgeist und ihr Ethos endlich auf ein zeitgemäßes Niveau bringen und bei einem offenen Wort nicht gleich Verrat wittern.

\section{Verfassungstreue}

Unter dem Aspekt der Verfassungstreue wäre eine andere Frage von Interesse, die sich freilich an M. adressiert, aber von Roellecke nicht gestellt wird. Würde er diese "Rechtsfrage « ernst nehmen, liefen seine Gleichungen "Beratung von Neonazis = illoyal/unkollegial" und "Veröffentlichung des Skandals durch Kollegen = illoyal/unkollegial « Gefahr, als unfreiwillige Komik gelesen zu werden. Zu fragen ist, ob Verfassungstreue, wie Anschütz es anläßlich seiner erzwungen-freiwilligen Emeritierung 1933 formulierte, Öffentlichrechtlern einen »hohen Grad innerlicher Verbundenheit... mit der Staatsordnung «, heute wäre zu sagen: Verfassungsordnung abverlangt.

Bei der Antwort ist Vorsicht geboten, will man dem BVerfG nicht auf seinen Abwegen zur Gesinnungstreue folgen oder die Öffentlichrechtler an das jeweilige Regime binden. Im Kontext des Grundgesetzes sind übermäßige Zweifel jedoch nicht angebracht: In diesem Staat sich »zu Hause fühlen«, nur regierungs- und staatstragende Oppositionsparteien beraten, stets ausgewogen urteilen - solche Treue mutet eine republikanisch-demokratische Verfassung auch denen nicht zu, die Verfassungsrecht lehren. Wohl aber, daß sie die Grundprinzipien einer demokratischen Republik nicht nur verkünden, sondern im Rahmen ihrer sonstigen professionellen Tätigkeit als Lehrer, Gutachter oder Berater auch praktizieren. Pro-fessor. Kein »klammheimlicher Faschismus « (Roellecke) in Theorie und Praxis, sondern Publizität und Transparenz. Studenten- und Kollegenschaft haben ein legitimes Interesse zu wissen, woran sie bei M. oder anderen sind. Wie hieß es doch anderswo: Sie predigten öffentlich Wasser und tranken heimlich Wein. Entgegen Roellecke, der sich auf ein Lob der moralischen Blindheit versteift, hat das nichts mit »Organisationserwartung für Grenzsituationen wie Außendarstellung, brauchbare Illegalität oder Rollenverflechtung « zu tun. Es geht vielmehr um die auch moralischen Erwartungen, die sich an (Hochschul-, Staatsrechts-)Lehrer richten. 